\title{
Tiefenbohrungen in den musikwissenschaftlichen Werk- und Autorschaftsbegriff mit digitalen Werkzeugen
}

\section{Einleitung}

„Musik ist wesentlich eine Aufführungskunst; ein Musikwerk realisiert sich in erster Linie in der Aufführung. "' Wie Andreas Münzmay und Christine Siegert in einem Aufsatz festhalten, ist diese Erkenntnis gar nicht so trivial, wie sie auf den ersten Blick vielleicht scheint. Sie hat vielmehr, wie die Autoren zeigen, erhebliche Auswirkungen auf den Werkbegriff und auf die Editionspraxis. Nicht das gedruckte Werk eines Komponisten oder einer Komponistin steht nach dieser Werkkonzeption im Mittelpunkt des Interesses, sondern die klingende Realisierung desselben. Zudem halten die Autoren fest, dass sich ,,in der Musikwissenschaft in den letzten Jahren im Anschluss an die allgemeine Geschichtswissenschaft ein Konzept etabliert hat, welches das kulturelle Handeln in den Mittelpunkt stellt. "2 Zuspitzend hatte bereits Johannes Kepper 2009 in seiner Dissertation herausgearbeitet, dass sich „Werkbegriff, Autorbegriff, das Konzept der Vollständigkeit, die Frage nach Autorintention, Autorisation und Authentizität und letztlich auch das herkömmliche Verständnis von Ausgaben allesamt als überkommen erweisen. " Modell des kulturellen Handelns, so heißt es in dem Aufsatz von Münzmay und Siegert weiter, ,als Gegenmodell zur autor- und opuszentrierten Geschichtsschreibung entworfen wurde, nimmt es nicht wunder, dass bislang noch kaum versucht wurde, diese Perspektive auf etablierte Komponisten des klassisch-romantischen Repertoires und deren Kompositionen zu übertragen oder es für editorische Konzepte fruchtbar zu machen.“ Die Autoren schlagen daher vor, auch „Aufführungsmateriale, Abschriften nach Drucken, instruktive Ausgaben und last but not least

\footnotetext{
${ }^{1}$ Andreas Münzmay, Christine Siegert: Phonographischer Text, Interpretation und Aufführungsmaterial als kritisch edierbarer Sachzusammenhang. Ein Beitrag zur Theorie der Edition von Klangdokumenten. In: editio 33, 20I9, S. IO-30, hier S. II.

2 Münzmay/Siegert 2019 (Anm. I), S. I5.

3 Johannes Kepper: Musikedition im Zeichen neuer Medien. Historische Entwicklung und gegenwärtige Perspektiven musikalischer Gesamtausgaben. Norderstedt 20II (Schriften des Instituts für Dokumentologie und Editorik. 5), S. I79; zit. nach Stefanie Acquavella-Rauch: (Musik)Edition im „,digitalen Zeitalter“ - Versuch einer Verortung konzeptioneller und struktureller Veränderungen. In: Symposiumsbericht Wege des Faches - Wege der Forschung? Hrsg. von Klaus Pietschmann. Mainz 20I6, https://schott-campus.com/wp-content/uploads/20I9/02/II5_Rauch.pdf, S. 7 (gesehen 3I.3.2O2I).
} 
Klangdokumente“ in die Edition eines Musikwerkes einzubeziehen. ${ }^{4}$ Martin Elste folgend, der schon in den I980er Jahren den Übergang von einer Partiturwissenschaft $\mathrm{zu}$ einer Klangwissenschaft gefordert und Methoden hierfür angemahnt hatte, ${ }^{5}$ geht es den Autoren also darum, auch die CD, also eine Tonaufnahme, als Werk zu begreifen und mittels der digitalen Edition zu erschließen.

Hier soll der Blick jedoch auf zwei andere Details dieser Überlegungen gerichtet werden, die meiner Meinung nach nicht weniger fruchtbar und untersuchenswert sind. Folgt man nämlich zum einen dem Gedanken, dass Musik kulturelles Handeln ist, geraten auch die Personen in den Blick, die an einer Musikaufführung beteiligt sind. Oder anders: Es geht auch um die Interpreten, die die klingende Realisierung eines Musikwerks vollziehen und eine Aufführung durch ihr Tun überhaupt erst möglich machen. Wie im Folgenden beleuchtet werden soll, ist eine (digitale) Sichtbarmachung dieses Tuns jedoch erst in Ansätzen geschehen. In Abschnitt 2 dieses Aufsatzes wird zu zeigen sein, welche Folgerungen dies für Regieklavierauszüge einer Interpretin hätte.

Und zum anderen lassen sich die vorstehenden Überlegungen Münzmays und Siegerts noch mit der folgenden Feststellung weiterdenken: Am Zustandekommen einer Tonaufnahme sind nicht nur die Musiker beteiligt, sondern auch Tonmeister und Toningenieur. Daher müsste auch die „Gruppenleistung Tonaufnahme“, 6 wie Hans-Joachim Maempel dieses Zusammenwirken treffend genannt hat, in einer Edition sichtbar gemacht werden.

\section{Digitale Sichtbarkeit: die Interpretin}

Verlagert man also den Fokus vom Werk und dem Komponisten oder der Komponistin auf das kulturelle Handeln, können auch Personen betrachtet werden, die längere Zeit nur als ,Nachschaffende“ bezeichnet wurden, nämlich die Interpreten und Interpretinnen. ${ }^{7}$ Hier sei zur Begriffsgeschichte nur kurz angemerkt, dass, wie Heinz von Loesch festhält, ,,im 20. Jahrhundert eine Fülle aufführungsbezogener Begriffe“ existierte, sich jedoch ,,in der zweiten Jahrhunderthälfte ,Interpretation“

4 Münzmay/Siegert 2019 (Anm. I), S. I5.

5 Martin Elste: Von der Partiturwissenschaft zu einer Klangwissenschaft. Überlegungen zur Schallplattenforschung. In: Jahrbuch des Staatlichen Instituts für Musikforschung Preußischer Kulturbesitz I983/84 [I987], S. II5-I44.

${ }^{6}$ Hans-Joachim Maempel: Technologie und Transformation. Aspekte des Umgangs mit Musikproduktions- und Übertragungstechnik. In: Musiksoziologie. Hrsg. von Helga de la Motte-Haber und Hans Neuhoff. Laaber 2007 (Handbuch der Systematischen Musikwissenschaft. 4), S. I60-I80, hier S. I63.

7 Vgl. hierzu etwa Heinz von Loesch: Autor - Werk - Interpret. In: Geschichte der musikalischen Interpretation im I9. und 20. Jahrhundert. Hrsg. von Thomas Ertelt und Heinz von Loesch. Bd. I: Ästhetik - Ideen. Kassel 20I9, S. 63-I27. 
als Zentralbegriff durchsetzte. “8 Und mit Münzmay/Siegert lässt sich das Folgende ergänzen:

Mit Sicherheit ließe sich trefflich und lange darüber streiten, was musikalische Interpretation überhaupt ist; einigen würde man sich vermutlich zumindest darauf, dass es sich gerade im 20. Jahrhundert um eine Kulturtechnik von größter Relevanz und Reichweite handelt, deren Erforschung, und hier gerade auch die philologische Aufarbeitung ihrer materialen Überlieferung, ohne Zweifel kulturhistorisch sinnvoll ist. Außerdem kann man guten Gewissens vielleicht doch zumindest behaupten, dass sich Interpretation sehr, sehr oft als künstlerische Auseinandersetzung mit einem bestimmten, als Aufführungsmaterial (oder zumindest als Proben- und Studienmaterial) benutzten Notentext (oder auch mehreren) darstellt $[\ldots] .{ }^{9}$

Es erscheint bereits bemerkenswert, dass sich - wie aus dem obigen Zitat ersichtlich ist - die Historische Musikwissenschaft anscheinend nach wie vor mit dem Begriff der Interpretation sehr schwer tut und dementsprechend wohl auch mit dem Begriff (oder der Tätigkeit und der Relevanz) des Interpreten bzw. der Interpretin. Dies überrascht schon insofern, als sich etwa Rüdiger Nutt-Kofoth in einem Aufsatz für einen ,,autorschaftsfernere[n], dafür medial orientierte[n] Werkbegriff“ stark gemacht hat und hierfür (am Beispiel von Strauss' Rosenkavalier, als ,,reale[ ] Ausprägung[] des pluriautorschaftlichen und mehrmedialen Werkes“") ausdrücklich auch die Oper einbezieht. Sichtbar wird an diesem Beispiel, wie der Autor zutreffend schreibt, ,dass die Werkkonstruktion auch der Oper nicht auf die ihr allemal schon inhärente medienübergreifende Text-Musik-Kombination beschränkt zu sein braucht, sondern zu ihr auch noch mediale Weiterungen gehören können“. Ausdrücklich hält der Autor zudem Folgendes fest: „Die Edition - würde sie dem hier veranschlagten Werk-Begriff folgen - müsste dann all diese Ausprägungen des Werkes zu ihrem Gegenstandsbereich rechnen. "10

\section{a. Die Interpretation als Werk, sui generis`}

Freilich ist auch in der Musikwissenschaft spätestens seit den Veröffentlichungen von Nicholas Cook $^{\text {II }}$ das Schlagwort der 'music as performance' bzw. 'work as performance' bekannt. In ihrem Kern richtet sich die Cook'sche These, so fasst es Heinz von Loesch zusammen,

\footnotetext{
8 Von Loesch 2019 (Anm. 7), S. I9.

9 Münzmay/Siegert 20I9 (Anm. I), S. 25.

${ }^{10}$ Rüdiger Nutt-Kofoth: Autorschaft, Werk, Medialität. Editionstheoretische Annäherungen an pluriautorschaftliche und plurimediale Werkkomplexe - mit einem germanistischen Blick auf das Phänomen Oper/Libretto. In: Perspektiven der Edition musikdramatischer Texte. Hrsg. von Thomas Betzwieser, Norbert Dubowy und Andreas Münzmay unter Mitarbeit von Markus Schneider. Berlin, Boston 2017 (Beihefte zu editio. 43), S. $25-38$, hier S. 34 und 36.

${ }^{I I}$ Nicholas Cook: Between Process and Product: Music and/as Performance. In: Music Theory Online 7,2 200I, https://mtosmt.org/issues/mto.oI.7.2/mto.oI.7.2.cook.html (gesehen 3I.3.202I); Nicholas Cook: Beyond the Score. Music as Performance. New York 20I3.
} 
gegen die Annahme eines Werkes vor bzw. jenseits der Aufführung. Musikalischer Sinn liege nicht im Werk selbst und nicht in den Intentionen des Autors. Und der Sinn sei auch nicht primär ein geistiger, mit Begriffen fassbarer hermeneutischer Sinn oder Struktursinn. Sinn werde vielmehr diskursiv - durch Rezeptionskontexte - gestiftet und vor allem durch den Interpreten. Und zwar im Rahmen eines performativen ,Aktes', einer 'real-time-performance', bei der der Körper und soziale Aktionen von ausschlaggebender Bedeutung seien: der Musiker, zwischen Musikern und Publikum sowie letztendlich von allen am Kulturereignis Musik Beteiligten. Die Partitur sei nicht mehr und nicht weniger als ein 'social script': ein Komplex von Handlungsanweisungen für eine Choreographie sozialer Interaktionen. ${ }^{\mathrm{I}}$

Insgesamt geht es Cook darum, den performativen Anteilen an einer Aufführung oder Interpretation eine größere Bedeutung zuzugestehen. Bereits im Abstract seines Textes heißt es:

The text-based orientation of traditional musicology and theory hampers thinking about music as a performance art. Music can be understood as both process and product, but it is the relationship between the two that defines 'performance' in the Western 'art' tradition. Drawing on interdisciplinary performance theory (particularly theatre studies, poetry reading, and ethnomusicology), I set out issues and outline approaches for the study of music as performance; by thinking of scores as 'scripts' rather than 'texts', I argue, we can understand performance as a generator of social meaning. ${ }^{13}$

Interessant für unseren Zusammenhang ist ferner - hierauf wird in Abschnitt 3 zurückzukommen sein -, dass Cook ,,auch Tonaufnahmen ein ästhetisches Daseinsrecht zu[gesteht], solange man sie auch als künstlerische ,Akte' und nicht als ,Texte“ begreift. “ ${ }^{14} \mathrm{Da}$ diese Reflexionen als geläufig vorausgesetzt werden dürfen, sei hier nur angefügt, dass sie bekanntlich auch in Deutschland auf große Zustimmung gestoßen sind und in den von verschiedenen Musik- und Theaterwissenschaftlern entwickelten performativitätstheoretischen Überlegungen sowie in den Diskussionen zur Performanz von Körper und Stimme breit rezipiert wurden. ${ }^{\text {Is }}$

Im Folgenden soll es vor diesem Hintergrund um die Interpretationsleistungen der Sängerin Anna Bahr-Mildenburg und die von ihr angefertigten Regieklavierauszüge gehen. Ich bin der Ansicht, dass diese als wichtige Dokumente aufgefasst werden müssen, die die Realisierung der Aufführung überhaupt erst sichtbar machen. Es handelt sich bei diesen Unterlagen um genau die ,medialen Weiterun-

\footnotetext{
${ }^{12}$ Von Loesch 2019 (Anm. 7), S. II5.

${ }^{13}$ Cook 200I (Anm. II), Abstract.

${ }^{14}$ Von Loesch 2019 (Anm. 7), S. II6.

${ }^{15}$ Vgl. etwa Erika Fischer-Lichte: Ästhetische Erfahrung. Das Semiotische und das Performative. Tübingen 200I; Christa Brüstle, Clemens Risi: Aufführungsanalyse und -interpretation. Positionen und Fragen der „Performance Studies“ aus musik- und theaterwissenschaftlicher Sicht. In: WerkWelten. Perspektiven der Interpretationsgeschichte. Hrsg. von Andreas Ballstaedt und Hans-Joachim Hinrichsen. Schliengen 2008, S. I08-I32; Hans-Joachim Hinrichsen: Musikalische Interpretation. Hans von Bülow. Stuttgart I999.
} 
gen“, für die sich Nutt-Kofoth in seinem oben genannten Aufsatz im Sinne eines medial orientierten Werkbegriffs stark machte. ${ }^{16}$

\section{b. Interpretationsmaterialien: Regieklavierauszüge von Anna Bahr-Mildenburg}

Bereits in meiner Dissertation ${ }^{17}$ konnte ich zeigen, dass die Regieunterlagen Anna Bahr-Mildenburgs zu Richard Wagners Ring des Nibelungen nicht nur ein Stück Aufführungsgeschichte dieses Werkes markieren, sondern gleichzeitig als performative Handlung zu verstehen sind. Für dieses Werk hat Anna Bahr-Mildenburg nicht nur Regieklavierauszüge, sondern auch Regiebücher für die Rolle der Brünnhilde in den Werkteilen Walküre, Siegfried und Götterdämmerung angefertigt; alle diese Dokumente blieben ungedruckt. ${ }^{18}$ Indem sie insbesondere in den letztgenannten Unterlagen verschriftlichte, was wie auf der Opernbühne zu sehen sein soll, nahm sie die performative (verstanden als mimisch-gestische) Dimension der Aufführung in den Blick. Die Regiebücher erscheinen daher als Möglichkeit, Körper und Stimme der Darstellerin als Elemente des Performativen zu analysieren, ja sie überhaupt erst einmal sichtbar zu machen. Ferner tut sich so die Möglichkeit auf, den Aufführungssinn ${ }^{19}$ der von Anna Bahr-Mildenburg verantworteten RingInszenierung $\mathrm{zu}$ entschlüsseln, die sie als alleinverantwortliche Regisseurin in der Spielzeit I92 I/22 in München auf die Bühne stellte. ${ }^{20}$

Auch für die Elektra von Richard Strauss gibt es zahlreiche interpretierende Eintragungen im Klavierauszug von Anna Bahr-Mildenburg, die in der Wiener Erstauffuhrung des Werkes im Jahr I909 die Rolle der Klytämnestra übernommen

\footnotetext{
${ }^{16}$ Nutt-Kofoth 2017 (Anm. I0), S. 34.

${ }^{17}$ Karin Martensen: Die Frau führt Regie. Anna Bahr-Mildenburg als Regisseurin des Ring des Nibelungen. Mit einem Anhang: Regiebücher zu Walküre, Siegfried und Götterdämmerung. München 2013 (Beiträge zur Kulturgeschichte der Musik. 7).

${ }^{\text {I8 }}$ Die drei genannten Regiebücher habe ich (in Abschrift) als Anlage zu meiner genannten Dissertation veröffentlicht.

19 Vgl. zum Begriff Hermann Danuser: Einleitung. In: Musikalische Interpretation. Hrsg. von dems. Laaber I992, S. 4-8. Danach gehören zum Aufführungssinn ,,sowohl die explizit formulierte Vortragsbezeichnung als auch die nicht notierten bzw. nicht notierbaren Textdimensionen“ (ebd., S. 4). Für den Aufführungssinn sei es wesentlich, dass er ,,in einer schriftlichen Textform vom Autor nicht genau, und schon gar nicht ein für alle Mal definiert werden kann. Seine Bedeutung ist abhängig von dem pragmatischen Kontext, in dem er sich realisiert“ (ebd., S. 4; beide Angaben zit. nach Stephan Mösch: Weihe, Werkstatt, Wirklichkeit. Wagners Parsifal in Bayreuth I882-I933. Kassel 2009, S. I9).

${ }^{20}$ Ferner fertigte Bahr-Mildenburg ein Regiebuch zu Wagners Parsifal mit Ausarbeitungen zu allen Hauptrollen an; dieses befindet sich unveröffentlicht beim Nachlass der Künstlerin im Theatermuseum Wien. Außerdem gibt es ein Regiebuch zur Figur der Ortrud in Wagners Lohengrin; dieses befindet sich ebenfalls unveröffentlicht bei ihrem Nachlass in Wien. Bahr-Mildenburgs Verlag, der ihr Regiebuch zu Tristan und Isolde im Druck veröffentlichte (Anna Bahr-Mildenburg: Tristan und Isolde. Darstellung des Werkes aus dem Geiste der Dichtung und Musik. Leipzig, Wien I936), kündigte zudem an, ein Regiebuch zu den Meistersingern von Nürnberg publizieren zu wollen. Dieses Vorhaben wurde aber nie verwirklicht; ein Regiebuch zu diesem Werk gibt es nicht.
} 
hatte. Dieser Klavierauszug soll im Folgenden im Mittelpunkt der Überlegungen stehen.

Bereits vor ihrem ersten Auftreten als Klytämnestra hatte sich Anna BahrMildenburg (I872-I947) Ruhm als hochdramatischer Sopran erworben. Ende I895 debütierte sie in Hamburg als Brünnhilde in Wagners Walküre. Bereits I897 gastierte sie zum ersten Mal als Kundry in Bayreuth. Ein Jahr später wurde sie auf Vermittlung Gustav Mahlers als Ensemblemitglied an die Wiener Hofoper verpflichtet. Dort trat sie als Ortrud in Wagners Lohengrin erstmals auf und feierte ihr Rollendebüt als Isolde in dessen Tristan und Isolde am I3. Februar I900. Nach dem Weggang Mahlers von der Wiener Bühne Ende 1907 gehörte die Darstellung der Klytämnestra zu Anna Bahr-Mildenburgs herausragendsten künstlerischen Erfolgen. I9I6 verabschiedete sie sich mit dieser Rolle von der Bühne der Wiener Hofoper. Nach dem Ende des Ersten Weltkrieges war sie nur noch sporadisch Gast an der Staatsoper (wie die Hofoper seitdem hieß), dann vor allem in der Rolle der Klytämnestra. Mit dieser Partie nahm sie I93 I bei den Augsburger Opernfestspielen ihren endgültigen Abschied vom öffentlichen Bühnenleben. ${ }^{21}$

Für ihre sängerischen und darstellerischen Leistungen erfuhr die Künstlerin in der zeitgenössischen Kritik geradezu hymnische Anerkennung. Von den Kritikern und von Mahler selbst wurde vor allem die Fähigkeit der Künstlerin herausgehoben, emotionalen Tiefgang der musikdramatischen Interpretation mit perfekter sängerischer Leistung zu verbinden. Auch mit ihrer Darstellung der Klytämnestra konnte sie bei Auffuhrungen in ganz Europa ${ }^{22}$ große Erfolge verbuchen. ${ }^{23}$

Für Bahr-Mildenburg ergab sich jedoch in der Vorbereitung der Klytämnestra das Problem, dass sie das Einverständnis oder auch nur die Unterstützung des Komponisten für die Konzeption dieser Rolle nicht (oder jedenfalls nicht in zufriedenstellender Weise) einholen konnte, obwohl sie sich stark darum bemühte. Tatsächlich geht aus Briefen der Künstlerin an ihren Ehemann Hermann

${ }^{21}$ Vgl. zu diesen biografischen Angaben Gustav Mahler: „Mein lieber Trotzkopf, meine süße Mohnblume“. Briefe an Anna von Mildenburg. Hrsg. und kommentiert von Franz Willnauer. Wien 2006, insbesondere S. 4I9-472.

${ }^{22}$ Die Londoner Premiere der Elektra fand mit Anna Bahr-Mildenburg als Klytämnestra am I9. Februar I9Io im Royal Opera House Convent Garden statt. Über den enormen Erfolg berichtete die Wiener Neue Freie Presse (mit Bezug auf die Berichterstattung in englischen Zeitungen) gleich zwei Mal in Superlativen: „,das größte musikalische Ereignis Englands seit vielen Jahren“ (NFP, 20.2.I9IO, S. I5); „ein Theaterereignis ersten Ranges“, es sei „noch niemals ein so schrankenloser Enthusiasmus“ erlebt worden (NFP, 24.2.I9IO, S. IO). Die Sängerin selbst berichtet über dieses Ereignis in ihren Erinnerungen (Londoner Erinnerungen. In: Anna Bahr-Mildenburg: Erinnerungen. Wien I92I, S. I59-I68) sowie in der NFP vom I6.4. I9II, S. I7f.

${ }^{23}$ In einer Rezension von Richard Specht hieß es etwa: „Die Mildenburg ist als Klytämnestra von grauenvoller tragischer Gewalt, einem beklemmenden Phantom gleich, von bösen Träumen gejagt, von tückischer Angst geschüttelt; ihre Schritte, die wie unter blutiger Last taumeln, ihre tastenden Hände, die nicht fassen können, was sie greifen wollen, ihre schweren Lider, auf denen die Müdigkeiten eines verwüsteten, grausamen Lebens lagern - eine Gestalt, wie sie die Opernbühne vor dieser großen Künstlerin kaum geahnt hat" (in: Die Musik, 2. Aprilheft, I908/o9, S. II2). 
$\mathrm{Bahr}^{24}$ aus der Zeit vor der Wiener Erstaufführung der Elektra hervor, dass es bei ihr bis zur Generalprobe des Werkes erhebliche Unsicherheiten über die Richtigkeit ihrer Interpretation gab. Am I9. Februar I909 etwa heißt es:

Wir arbeiten also mit Strauss! [...] Ob ihm meine Klytämnestra recht ist, hab ich eigentlich nicht herausgebracht. Fast hab ich das Gefühl, dass er mehr oder Anderes erwartet hat. Meine Leistung ist ja noch total unfertig und dann glaube ich, dass ich ihm zu wenig verblödet und krankhaft die Rolle auffasse. Wenn er mich auf der Bühne sehen würde mit allem, was drum und dran ist, würde er mir ganz sicher recht geben und mich verstehen. Leider kommt er nicht zur Aufführung, weil er in Italien ist. ${ }^{25}$

Ausweislich der zwischen Hermann Bahr und Richard Strauss gewechselten Briefe war Letzterer nach der Erstaufführung der Elektra in Berlin, die am Is. Februar I909 stattfand, umgehend weiter nach Wien gereist und traf dort am I7. Februar ein. ${ }^{26}$ Seinen Aufenthalt in Wien unterbrach er aber für I4 Tage, um nach Garmisch bzw. München (und möglicherweise auch nach Mailand) zu reisen. Am I6. März war er wohl wieder in Wien. ${ }^{27}$ Insofern ist es kein Widerspruch, wenn Bahr-Mildenburg nach der öffentlichen Generalprobe der Elektra am 22. März, die für die Sängerin ein großer Erfolg war, an ihren Mann schreibt: „Strauss kommt und der soll einen vollen Eindruck haben, wenn ich die Klytämnestra darstelle. Ich gestalte sie mir innerlich immer mehr aus und die Figur klärt sich in mir immer mehr. "28 Man kann daraus schließen, dass auch bis dahin keine für die Künstlerin zufriedenstellende Verabredung zur Umsetzung der Klytämnestra zustande gekommen war.

Wie es wohl bei allen bis dahin entstandenen Werken seine Gewohnheit war, reiste Strauss nur für Uraufführungen im Vorfeld an, um mit den Sängern und Sängerinnen ,,auf den Ausdruck hin“ zu studieren. ${ }^{29}$ Alles übrige wollte er, wie

${ }^{24}$ Anna von Mildenburg und Hermann Bahr heirateten am 22. August I909; die Künstlerin nannte sich von da an Bahr-Mildenburg.

${ }^{25}$ Theatermuseum Wien, Nachlass Anna Bahr-Mildenburg, Karton 46 (Briefe Anna Bahr-Mildenburg an Hermann Bahr der Jahre I909 und I9Io).

${ }^{26} \mathrm{Vgl}$. Brief von Strauss an Bahr vom I2. Februar I909; Richard Strauss im Briefwechsel mit Hans Sommer, Hermann Bahr und Willy Levin. Mit ergänzenden Korrespondenzen von Pauline de AhnaStrauss, Antonie Sommer, Anna Bahr-Mildenburg und Franz Strauss. Hrsg. von Christian Cöster. Mainz 2019, S. I96.

${ }^{27}$ Vgl. Brief von Bahr an Strauss vom I6.3.I909; Strauss-Briefwechsel 2019 (Anm. 26), S. I97.

${ }_{28}$ Theatermuseum Wien, Nachlass Anna Bahr-Mildenburg, Karton 46 (Briefe Anna Bahr-Mildenburg an Hermann Bahr der Jahre I909 und I9I0).

${ }^{29}$ Vgl. dazu den Brief von Strauss an Schuch vom I5. November I9Io, in dem es um Strauss' Anwesenheit in Dresden für eine Probe des Rosenkavalier ging, dessen Uraufführung am 26. Januar I9II stattfand: ,Aller Voraussicht nach werde ich am II. Dezember Abends 7 Uhr in Dresden ankommen und kann bis I2. Dezember abends da bleiben, natürlich wenn ich in diesen 2 Tagen eine vollständige Klavierprobe des Rosencavalier anhören kann. Ich darf wohl annehmen, daß der I u. II. Akt bis dahin bereits auswendig u. d. III. Akt von Noten wenigstens so correkt gesungen werden kann, daß man mit den Sängern bereits auf den Ausdruck hin studieren kann. Sonst nämlich hat meine Anwesenheit keinen Zweck“; Richard Strauss - Ernst von Schuch: Ein Briefwechsel. Hrsg. von Gabriella Hanke 
dies bei anderen Einstudierungen auch geschah, Regisseur und Dirigent vor Ort überlassen. ${ }^{30}$ Der Briefwechsel zwischen Bahr und Strauss belegt jedoch, dass es in Bezug auf die Elektra-Aufführung in Wien erhebliche Querelen gab, die nicht nur aus Besetzungsschwierigkeiten in Bezug auf die Rolle der Elektra resultierten, sondern auch daraus, dass der Dirigent Felix von Weingartner (der als Nachfolger Mahlers die Leitung der Wiener Hofoper übernommen hatte) die Aufführungen der Elektra nur halbherzig betrieb. ${ }^{31}$ Dies spiegelt sich auch in den Briefen BahrMildenburgs an ihren Ehemann wider und wird möglicherweise zu ihrer Verunsicherung in Bezug auf die Rolle der Klytämnestra beigetragen haben. - Auch der Librettist Hugo von Hofmannsthal war kein rechtzeitig erreichbarer Ansprechpartner für die Künstler: Zur Uraufführung der Elektra (die am 25. Januar I909 stattfand) ${ }^{32}$ reiste er auf Strauss' Bitte Mitte Januar nach Dresden, um dort „,nach dem Rechten zu sehen“. ${ }^{33}$ In Wien traf Hofmannsthal erst zehn Tage vor der Aufführung ein.

Hieraus wird man freilich nicht schließen dürfen, dass Strauss und Hofmannsthal die gesangliche und dramatisch-gestische Umsetzung ihrer Figuren auf der Bühne gleichgültig war. Vielmehr wollte Strauss offenbar Auktorialität im Hinblick auf die Umsetzung seiner Figuren nicht in Anspruch nehmen. Es verwundert daher nicht, dass Bahr-Mildenburg von Strauss keinerlei Hinweise zu der Frage bekam, was in der Partitur möglicherweise in Bezug auf die gestische Ausgestaltung zu ,entdecken' sei, da er dies seinen Künstlern nicht vorschreiben, sondern ihrer jeweils eigenen Gestaltungskraft überlassen wollte. Es verwundert auch nicht, dass Bahr-Mildenburg die Arbeit an der Figur auf der Grundlage ihrer Notizen im Klavierauszug ganz allein leistete, und zwar so, wie sie es selbst für richtig hielt.

Für den Erarbeitungsprozess dieser Rolle kann man also nicht davon ausgehen, dass dieser von Strauss in dem Maße mitgetragen wurde, wie es bei den Liedern des Komponisten der Fall war, die er mit seiner Ehefrau erarbeitete (vgl. dazu die Ausführungen unten). Doch war Strauss bekanntlich von der Interpretation der Klytämnestra durch Bahr-Mildenburg so begeistert, dass er ihr das Skizzenbuch zur Elektra zum Geschenk machte. Dort findet sich folgende Widmung für die Künstlerin: „Der genialen Darstellerin der Klytämnestra in Wien[,] Frl. von Milden-

Knaus. Berlin I999 (Veröffentlichungen der Richard-Strauss-Gesellschaft. I6), S. I6I. - Für den I6. Januar I9II kündigte Strauss sein abermaliges Kommen an; ebd., S. I72.

${ }^{30}$ Für den Rosenkavalier ist davon die Rede, dass Strauss mit Ausnahme der o.g. Probe die Arrangierproben Max Reinhardt, Hugo von Hofmannsthal und Alfred Roller überlassen wollte; StraussSchuch-Briefwechsel i999 (Anm. 29), S. I65.

${ }^{31} \mathrm{Vgl}$. Strauss-Briefwechsel 2019 (Anm. 26), S. I99f.

${ }^{32}$ Hermann Bahr war bei der Uraufführung der Elektra in Dresden anwesend und schrieb hierüber eine Rezension, die am 26.I.I909 in der Wiener Neuen Freien Presse erschien.

${ }^{33}$ Richard Strauss - Hugo von Hofmannsthal. Briefwechsel. Hrsg. von Willi Schuh. München I990, S. 52 . 
burg[,] zur freundlichen Erinnerung an ihren größten Bewunderer. Der dankbare Componist der Elektra. Dr. Richard Strauss, Garmisch, 7. Mai I909“.34 Und wie zu zeigen sein wird, kann man anhand der Interpretationshinweise, die die Künstlerin in ihrem Klavierauszug vermerkte, über ihre Werkauffassung (und damit auch über das Werk selbst) eine Menge lernen. ${ }^{35}$

In ihren Interpretationsnotizen im Elektra-Klavierauszug hat sich Anna BahrMildenburg auch mit der psychischen Befindlichkeit der Klytämnestra beschäftigt. Besonders bemerkenswert ist die Musik- und Textausdeutung der Sängerin in der Szene zwischen Elektra und Klytämnestra, in der Letztere von ihren furchtbaren Träumen erzählt: Bereits die erste Frage, die Klytämnestra an Elektra richtet (,,Weißt du kein Mittel gegen Träume“) ist nur vordergründig freundlich; die Musik mit ihren überaus vieldeutigen Akkorden (zwei Takte nach Ziffer I78) deutet hingegen an, dass es natürlich keine angenehmen Träume sein werden, von denen dann im Anschluss die Rede ist. Bahr-Mildenburg notiert zu diesen beiden Takten: „lächeln wollen aber dabei schaudern" ${ }^{36}$

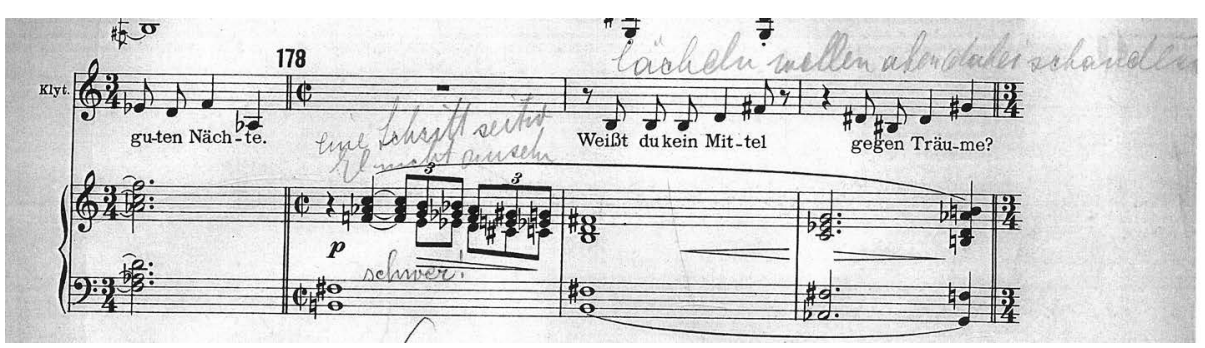

Abb. I: Elektra, Klavierauszug, S. 77, Ausschnitt.

Zu den Worten Klytämnestras an Elektra „du könntest vieles sagen, was mir nützt“ hat die Sängerin sowohl das im Klavierauszug abgedruckte ,espressivo“ mit Bleistift eingekreist als auch den Einsatz des ersten Horns mit einer Ziffer I markiert, mit dessen ,befremdlichen' Tönen die zuvor ,rauschhafte‘ Musik abbricht. Ihre

${ }^{34}$ Theatermuseum Wien, Sign.: HS_Vk904BaM.

${ }^{35}$ Vgl. hierzu Karin Martensen: Singen und Darstellen - Autorschaft und Macht auf der Bühne. Über die Zusammenarbeit von Anna Bahr-Mildenburg und Richard Strauss bei dessen Elektra. In: Studien zur Musikwissenschaft. Beihefte der Denkmäler der Tonkunst in Österreich 59, 20I7, S. I2I-I40; Dies.: Der annotierte Klavierauszug - eine Quelle für die Interpretationsforschung? In: Die Musikforschung 2016, H. I, S. 25-45; Dies.: Bühnenkostüm und Klavierauszug als Charakterstudien: Überlegungen zu Anna Bahr-Mildenburg als Klytämnestra in Richard Strauss' Elektra. In: „,Worte klingen, Töne sprechen" - Richard Strauss und die Oper. Symposium anlässlich der Richard Strauss-Ausstellung im Theatermuseum Wien, 22.-23. Jänner 20I5. Hrsg. von Christiane Mühlegger-Henhapel und Alexandra Steiner-Strauss. Wien 2015, S. 39-47.

${ }^{36}$ Klavierauszug Elektra, S. 77 (Text von Otto Singer, Berlin, PlattenNr. A 5654 F. In der Österreichischen Nationalbibliothek Wien wird der Druck unter der Signatur MS67375-4 ${ }^{\circ}$ aufbewahrt). Alle Transkriptionen erfolgen zeichengenau. 
Anweisung an Klytämnestra zu diesen Worten lautet, dass sie „,schläfrig u. blöd“37 klingen müssten..$^{8}$

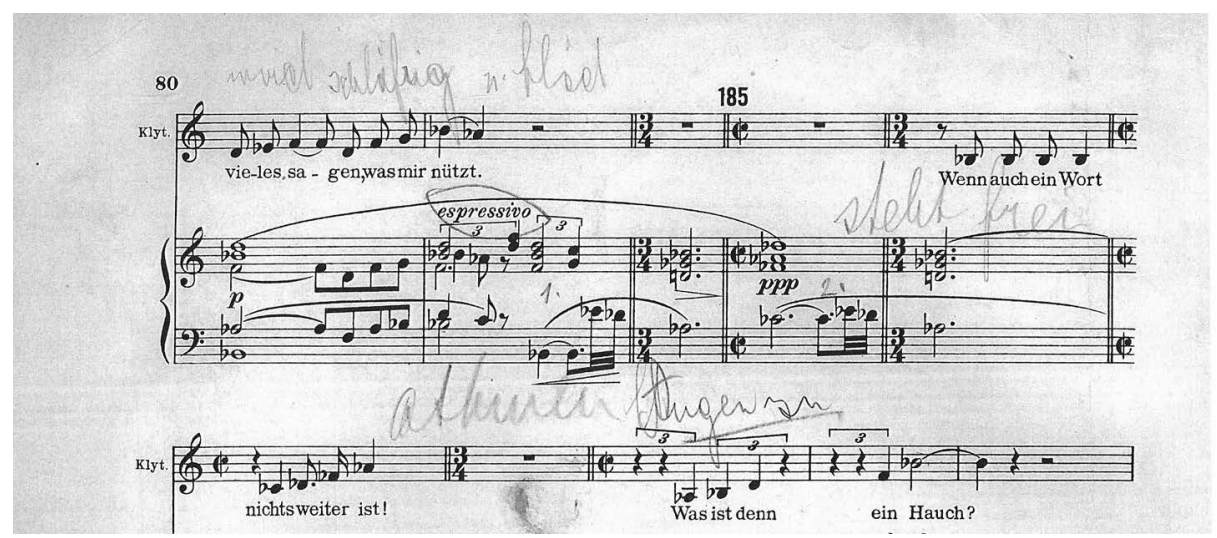

Abb. 2: Elektra, Klavierauszug, S. 80, Ausschnitt.

Und auch in den Worten ,ich bin nicht einmal krank“ (in der die Geigen mit einer sfz-Anweisung quasi in die Tiefe abstürzen) hört die Künstlerin Ausbrüche des geistigen Wahns Klytämnestras (vgl. dazu die Ausführungen in Anm. 37). Die Anweisung lautet: „,blöd“, ,furcht gepeinigt““. 39

Durch ihre genannten Anweisungen konnte Bahr-Mildenburg die skizzierten Spannungsverhältnisse zwischen Text und Musik gestisch sichtbar machen. Hierfür nutzte sie, wie hier nur kurz angedeutet werden kann, nicht nur die Musik, sondern vor allem den Text des Werkes, wie sie ihn im Klavierauszug vorfand. Dies betrifft etwa ihren Eintrag zum Text „,die mir das Blut aussaugen“; hier heißt es: „saugend im Ton“. ${ }^{40}$ - In meiner Dissertation über die Inszenierung Bahr-Mildenburgs von Wagners Ring des Nibelungen konnte gezeigt werden, dass sie auch für ihre Darstellung der Brünnhilde vor allem den subjektiv-emotionalen Gehalt nutzte, der dem

\footnotetext{
${ }^{37}$ Es ist nicht leicht, eine angemessene ,Übersetzung“ des Wortes „,blöd“ zu finden. Zwar liegt es auf der Hand, dass hier nicht die heutige Bedeutung dieses Wortes als ,albern, dumm, einfältig“" gemeint sein kann (vgl. Duden, https://www.duden.de/rechtschreibung/bloed; gesehen 6.I.2O2I). Die mittelhochdeutsche Bedeutung von „blœde“ war ,schwach, zart“, die althochdeutsche Bedeutung von „,blōdi“ hingegen „unwissend, scheu“ (vgl. ebd.). Bis zum Ende des I9. Jahrhunderts ist zudem die Wortbedeutung ,schüchtern, furchtsam“ zu finden (Hermann Paul: Deutsches Wörterbuch. Bedeutungsgeschichte und Aufbau unseres Wortschatzes. Io., überarb. und erweiterte Aufl. von Helmut Henne, Heidrun Kämper und Georg Objartel. Tübingen 2002, S. I79). Angesichts der geistigen Verfassung Klytämnestras insgesamt hielte ich es aber auch nicht für ausgeschlossen, dass hier die Bedeutung ,wahnhaft-krank“ gemeint ist.

${ }^{38}$ Klavierauszug Elektra (Anm. 36), S. 80.

${ }^{39}$ Klavierauszug Elektra (Anm. 36), S. 82.

${ }^{40}$ Klavierauszug Elektra (Anm. 36), S. 70.
} 


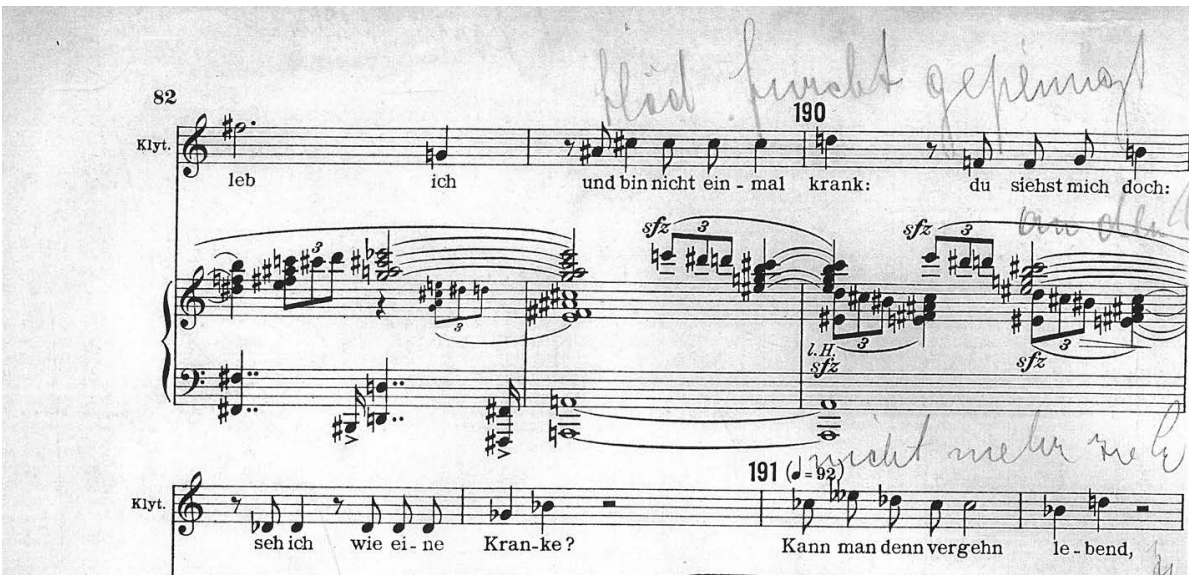

Abb. 3: Elektra, Klavierauszug, S. 82, Ausschnitt.

Text/dem Libretto in ihren Augen innewohnte. Die Musik der drei Ring-Werkteile hingegen nutzte sie nur als ,Anker“ bzw. sah sie als Impuls für die emotionale Haltung der Figuren an..$^{41}$

Ein starkes Indiz hierfür lässt sich auch im Vergleich des Mildenburg'schen Klavierauszugs mit dem ihrer Kollegin Ernestine Schumann-Heink (I86I-I936) aufzeigen. Diese war zwischen I883 und I897 an der Hamburger Oper engagiert und lernte Anna Bahr-Mildenburg dort kennen. Im Januar I909 übernahm SchumannHeink in der Dresdner Uraufführung der Elektra unter der Leitung von Ernst von Schuch die Rolle der Klytämnestra. Es handelt sich daher um eine Vorstellung, die nur zwei Monate vor der Wiener Aufführung mit Anna Bahr-Mildenburg in derselben Rolle stattfand.

Zwar versah auch Schumann-Heink ihren Klavierauszug der Elektra mit handschriftlichen Eintragungen zur Figur der Klytämnestra, doch beschränkte sie sich dabei auf solche, die die korrekte, ausdruckshafte Deklamation des Textes sicherstellen sollten. Daher finden sich in ihrem Auszug, der sich heute in der Claremont University in Kalifornien befindet, ${ }^{42}$ beinahe in jedem Takt Zählzeichen, ferner einige Wortunterstreichungen, Crescendogabeln und Atemzeichen. Gelegentliche wortsprachliche Eintragungen wie ,aufpassen“ dienten laut Stephan Lowell Moss der Abstimmung zwischen Schumann-Heink und ihrer Klavierbegleiterin, mit

\footnotetext{
${ }^{41}$ Vgl. Martensen 20I3 (Anm. I7), S. 236-242.

${ }^{42}$ Dies ist vermutlich dadurch zu erklären, dass Schumann-Heink seit 1908 amerikanische Staatsbürgerin war und später in Hollywood/USA verstarb. - Für den Hinweis auf den Klavierauszug und die Dissertation von Stephan Lowell Moss (Ernestine Schumann-Heink as Klytemnestra in the Premiere of Elektra: An Analysis of Annotations in Her Working Scores. The Claremont Graduate School, I989), die sich gleichfalls in der Bibliothek der Claremont University befindet, bedanke ich mich herzlich bei Alexander Erhardt (Mitherausgeber der Elektra in der Richard-Strauss-Gesamtausgabe).
} 
der sie die Rolle einstudierte. ${ }^{43}$ Zugleich dürfte die Künstlerin sie als Hilfestellung angesichts der schwierigen rhythmischen Verhältnisse in der Partie genutzt haben. Eintragungen hinsichtlich der dramatischen Ausgestaltung der Figur gibt es jedoch nicht. Schumann-Heink konzentrierte sich vielmehr auf die Ausgestaltung des Textes. Ihre Intention war es - so formuliert es zumindest Stephan Lowell Moss "to project the verbal content of the libretto clearly, and to carry the emotional horror and intensity to the audience." ${ }^{44}$

Wie man im Vergleich der beiden Klavierauszüge erkennen kann, sind die Herangehensweisen der beiden Künstlerinnen durchaus verschieden. Auch wenn man aus diesen Unterlagen natürlich nicht auf spätere Aufführungen schließen kann, steht jedenfalls im Falle von Anna Bahr-Mildenburg fest, dass ihre Interpretation der Klytämnestra vom starken Wohlwollen des Komponisten getragen war. Zudem ist dieser Klavierauszug aus vielerlei Gründen von hohem theatergeschichtlichem Wert: Er reflektiert die fortschrittlichen Bestrebungen hinsichtlich der gestischen Ausgestaltung der Figuren, die seit der Jahrhundertwende ganz allmählich auf der Bühne Raum gewannen, und zeigt die Künstlerin als Fahnenträgerin dieser neuen Denkungsarten. Sie entsprach damit zugleich den Vorstellungen des Komponisten, der sich nicht, gewöhnliche Opernsänger' für seine Werke wünschte, sondern - wie man heute sagen würde - Sängerdarsteller. ${ }^{45}$ Wissenschaftlich interessant erscheint auch, dass mit Hilfe der Einzeichnungen Anna Bahr-Mildenburgs in ihrem Elektra-Klavierauszug die Medialität von Körper und Singstimme der Darsteller und Darstellerinnen im Musiktheater auch für die Forschung nutzbar gemacht werden kann. Diese Einzeichnungen beschreiben nämlich, wie Emotion auf der Bühne sichtbar wird. Und sie zeigen, dass Musikmachen eine soziale Praxis ist.

Es könnte daher eine Bereicherung für die Interpretationsforschung und für die digitale Musikwissenschaft sein, wenn man die Interpretationsleistung BahrMildenburgs als Werk ,sui generis' auffassen würde. Diese kann aber nur sichtbar gemacht werden, wenn man ihre Regieklavierauszüge mit digitalen Methoden erarbeiten und präsentieren würde. Wie dies geschehen und von welchen bereits geleisteten Arbeiten man hierfür profitieren könnte, soll nachstehend dargelegt werden.

\section{c. Sichtbarkeit der Interpretation in einer Edition}

Es stellt sich also die Frage, wie das Werk der Interpretin bzw. des Interpreten sichtbar gemacht und womöglich in Zusammenhang mit anderen Aufführungspraktiken

\footnotetext{
${ }^{43}$ Vgl. etwa Klavierauszug Schumann-Heink, S. 67, zum Text „was ist wahr und was ist Lüge“. - Bei Anna Bahr-Mildenburg gibt es solche Eintragungen nicht.

${ }^{44}$ Lowell Moss 1989 (Anm. 42), S. 89.

${ }^{45}$ Vgl. dazu Karin Martensen: Die Isolde einer ganzen Generation: Anna Bahr-Mildenburg. In: wagnerspectrum 20I4, H. 2: Schwerpunkt: Wagner und die bildende Kunst, S. 233-262.
} 
bzw. -materialien gestellt werden kann. In Bezug auf den Nachholbedarf in der Musik- bzw. in der dort etablierten Editionswissenschaft hält Stefanie AcquavellaRauch fest, dass struktureller Veränderungsbedarf bestehe:

So sehr inzwischen ausgehend von der Literaturwissenschaft in der musikalischen Biographik, Editorik sowie grundsätzlich über Begrifflichkeiten wie ,Autor' und ,Werk nachgedacht wird und so sehr ein generelles Umdenken innerhalb der Disziplin Musikwissenschaft in den 20ooer und 20I0er Jahren feststellbar ist, umso erstaunlicher ist es, dass die Rolle der Editor_innen für die Musikedition bisher nicht hinterfragt wurde, sondern nahezu vollständig ausgeblendet zu werden scheint. Es ist an der Zeit, die inzwischen lang zurückreichende Autorschaftsdebatte in diese Richtung zu erweitern. ${ }^{46}$

Dies gilt allerdings nicht nur für Editorinnen und Editoren, sondern auch für Interpreten eines Werkes. Wie mir scheint, beschäftigen sich Editionsprojekte zwar mit unterschiedlicher Schwerpunktsetzung mit Komponisten und deren Werkprozess. ${ }^{47}$ Von anderen Quellenarten jedoch als dem vorhandenen Skizzen- und Entwurfsmaterial eines Werkes, nämlich etwa Libretto, Interpretationshinweisen, Regiebüchern etc., „,berücksichtigen Ausgaben mit eher herkömmlicher methodischer Ausrichtung in der Edition maximal das Libretto, das teilweise aber nicht einmal separat abgedruckt, sondern gleichsam als Bestandteil der Musik, mitediert ${ }^{6}$ oder als Faksimile beigegeben wird. Anderes Material wird dabei zwar natürlich ebenfalls zur Kenntnis genommen, fließt aber traditionellerweise entweder in das Vorwort oder den Kritischen Bericht und nur selten in die Edition ein bzw. wird mit ediert"..$^{8}$

Drei Ausnahmen sind hier zu nennen, obwohl hierbei sogleich Einschränkungen gemacht werden müssen. So setzt sich das Detmolder Hoftheater-Projekt ${ }^{49}$ erstmals im Detail mit den überlieferten Quellen aus der Blütezeit des Detmolder Hoftheaters auseinander. Das Projekt hat sich somit zur Aufgabe gemacht, neben den Aufführungsmaterialien des Hoftheaters die unterschiedlichsten Kontextmaterialien zu erschließen, so dass ein erster Überblick gegeben werden kann, welche auf die Aufführung bezüglichen Materialien überliefert sind und welchen Informationsgehalt diese haben. ${ }^{50}$ Ziel ist es, ,,die Beziehungen der Objekte in den Mittelpunkt zu rücken“. Daher werden ,,alle Materialien, die zum Bereich ,Hoftheater gehören, erfasst und so verknüpft, dass die Informationen über die Objektgrenzen

\footnotetext{
${ }^{46}$ Acquavella-Rauch 2016 (Anm. 3), S. 7.

${ }^{47} \mathrm{Vgl}$. etwa die Auflistung bei Acquavella-Rauch 2016 (Anm. 3), S. 2-6.

${ }^{8}$ Acquavella-Rauch 2016 (Anm. 3), S. 4.

${ }^{49}$ Der vollständige Projekttitel lautet: Entwicklung eines MEI- und TEI-basierten Modells kontextueller Tiefenerschließung von Musikalienbeständen am Beispiel des Detmolder Hoftheaters im I9. Jahrhundert (I825-I875); http://www.hoftheater-detmold.de (gesehen 3I.3.202I).

${ }^{\text {so }}$ Vgl. Irmlind Capelle: Quellen zur Auffuihrung - Beispiele aus dem Detmolder Hoftheater-Projekt. In: Aufführung und Edition. Hrsg. von Thomas Betzwieser und Markus Schneider. Berlin, Boston 2019 (Beihefte zu editio. 46), S. III-I24.
} 
hinweg abgefragt werden können. “si Doch, wie Irmlind Capelle berichtet, sind „,[s]ogenannte Regiebücher, also durchschossene handschriftliche oder gedruckte Textbücher, bei denen auf den leeren Blättern Anmerkungen und Skizzen zur Szene vermerkt sind, bisher in den Materialien nicht nachgewiesen. Nach unserem gegenwärtigen Wissensstand enthalten lediglich einige gewöhnliche Textbücher und der Klavierauszug zu Fra Diavolo wenige Regieanmerkungen."s2 Auch wenn in diesem Projekt durch die kontextuelle Tiefenerschließung aller übrigen Materialien mit MEI und TEI ${ }^{53}$ ein bedeutender Schritt gemacht ist, muss auch über die Einbeziehung von Regiebüchern nachgedacht werden. Wie diese Materialien in den Kontext eingearbeitet werden könnten, und insbesondere die Frage, was ein Regiebuch für das Verständnis des jeweiligen Werkes bedeuten könnte, muss daher für den Augenblick offenbleiben.

Das Projekt OPERA - Spektrum des europäischen Musiktheaters in Einzeleditionen, seit Januar 20I3 an der Goethe-Universität Frankfurt am Main (zuvor an der Universität Bayreuth) angesiedelt, hat Anfang 2009 seine Arbeit unter der Trägerschaft der Akademie der Wissenschaften und Literatur in Mainz aufgenommen. Laut seiner Website hat das Projekt ,,die kritische Edition herausragender Werke des europäischen Musiktheaters in exemplarischen Einzelausgaben zum Ziel. “ Die Auswahl der zu edierenden Kompositionen ist an die musiktheatergeschichtliche Bedeutung des jeweiligen Werkes sowie die mit der jeweiligen Komposition verbundene editorische Problemstellung gebunden. ${ }^{54} \mathrm{Im}$ Modul 3 des Projekts mit dem Titel „Aufführungspraxis und Interpretation“ befinden sich Henrico Leone von Agostino Steffani, Leonora ossia L'amor conjugale von Ferdinando Paër und Der Vogelhändler von Carl Zeller. Mit diesen Werken soll „,das Problemfeld Interpretation unter ganz unterschiedlichen Gesichtspunkten“ thematisiert werden. „Beispielsweise vermag Paërs Oper mit dem Phänomen von aufführungsbedingten Veränderungen ein bedeutsames und editorisch heikles Problem der Opernpraxis zu repräsentieren. Bei der Operette hingegen liegen auf den Interpreten zurückgehende textliche

\footnotetext{
${ }^{51}$ Irmlind Capelle: Die Bestände des Lippischen Hoftheaters Detmold (I825-I875). Durch digital gestützte Tiefenerschließung zu neuen Forschungsmöglichkeiten. In: Beitragsarchiv zur Jahrestagung der Gesellschaft für Musikforschung Halle/Saale 20I5. Hrsg. von Wolfgang Auhagen und Wolfgang Hirschmann. Mainz 20I6, https://schott-campus.com/die-bestaende-des-lippischen-hoftheaters/, urn:nbn:de:Ior:I-20I609I3I284, S. I-8, hier S. I (gesehen 3I.3.202 I).

${ }_{52}$ Capelle 2019 (Anm. 50), S. II3.

${ }_{53}$ Vgl. Capelle 2016 (Anm. 5I). - Capelle erläutert überdies, dass auf diese Weise ein ,großer gemeinsamer Wissensraum zum Thema ,Detmolder Hoftheater ' [entsteht], der zudem jederzeit erweiterbar ist. Die Bereitstellung eines solchen Wissensraums statt der eindimensionalen Erschließung eines Bestandes erleichtert grundsätzlich die Forschung. Musste man bisher die unterschiedlichen Bereiche mühsam selbst ,durchforsten' und scheiterte man dabei häufig an der Materialfülle, so werden jetzt die Grenzen durchlässig: Auch beiläufige Informationen werden abrufbar und erweitern bzw. präzisieren das Wissen z. B. über eine Person“; Capelle 2016 (Anm. 5I), S. 4 .

${ }^{54}$ http://www.opera.adwmainz.de/das-projekt.html (gesehen 3I.3.202I).
} 
Interpolationen vor, welche in einigen Fällen auch die musikalische Seite nicht unerheblich tangieren. " 55

Wie es scheint, werden Aufführungsbedingungen damit nur insoweit berücksichtigt, als sie tatsächlich Veränderungen der Musik nach sich gezogen haben. Was aber, wenn dies vom Interpreten nicht beabsichtigt war und Eingriffe in den Text tatsächlich nicht vorliegen, aber die Interpretation dennoch durch Materialien belegt und zudem als Meilenstein in der Aufführungsgeschichte eines Werkes bezeichnet werden kann, wie eben im Fall von Bahr-Mildenburgs kommentiertem Klavierauszug zur Elektra?

Das 20I I begonnene Forschungsprojekt Kritische Ausgabe der Werke von Richard Strauss widmet sich dessen Kompositionen mit den Mitteln moderner Editionsphilologie. Das Projekt verfolgt das Ziel, sämtliche Bühnenwerke, genuine Orchesterwerke, Lieder und Gesänge sowie kammermusikalische Werke mit den modernen Methoden historisch-kritischer Editionstechnik in neu gesetzten Partituren mit kritischem Apparat vorzulegen. Auf diese Weise sollen authentische Werktexte verfügbar werden, die von Schreibfehlern, Kopisten- und Stichfehlern sowie von nicht durch den Komponisten autorisierten Zusätzen bereinigt sind und den Strauss'schen Intentionen so nahe wie möglich kommen, auch in der Darstellungsweise. $^{56}$

$\mathrm{Zu}$ Strauss` Liedern mit Klavierbegleitung op. Io bis op. 29 (erschienen als Band II/2 der Serie II „Lieder und Gesänge für eine Singstimme“) schreibt der Bandherausgeber Andreas Pernpeintner in der Einleitung, dass hierfür auch Eintragungen in den Handexemplaren von Pauline de Ahna-Strauss berücksichtigt wurden. Der Komponist führte seine Lieder häufig zusammen mit seiner Ehefrau Pauline auf. Der Herausgeber erläutert hierzu:

Die Eintragungen umfassen Vermerke im Sinne einer Aufführungshilfe (z.B. Atemzeichen), aber auch Modifikationen des Notentexts (z.B. versetzte Dynamikangaben, selten gar geänderte Noten der Singstimme) sowie ab und an Fehlerkorrekturen. Wohlgemerkt: Derartige Eintragungen stammen oft vom Komponisten selbst oder wurden jedenfalls von ihm als dem Klavierbegleiter mitgetragen. Sie werden deshalb in der Kritischen Richard-Strauss-Ausgabe dokumentiert, allerdings im Regelfall nicht so interpretiert, als lägen hier Eingriffe in die gültige Werkgestalt vor. Manche dieser Eintragungen können heutigen Interpreten als Anregung dienen, andere Vermerke (gerade die erstaunlich häufigen Atemzeichen) weisen eher auf damals übliche, heute veraltet wirkende Vortragsweisen oder speziell auf stimmliche Eigenarten von Pauline Strauss hin..$^{57}$

\footnotetext{
${ }^{55}$ Alle Zitate: http://www.opera.adwmainz.de/werkauswahl.html (gesehen 23.I0.2020).

${ }^{56} \mathrm{Vgl}$. https://www.richard-strauss-ausgabe.de/vorwort/ (gesehen 4. I0.2020).

${ }^{57}$ https://www.richard-strauss-ausgabe.de/band/?volume=b40I06\&chapter=el (gesehen 4. I0.2020).
} 
Ferner erläutert der Herausgeber, dass ,,[s]ubstanzielle Vermerke (beispielsweise Dynamikangaben und ausformulierte Vortragsbezeichnungen) im neuedierten Notentext in spitzen Klammern wiedergegeben“"werden.

Vor dem Hintergrund dieses Editionskonzepts erklärt sich, dass weder die Eintragungen Anna Bahr-Mildenburgs in ihren Klavierauszug noch die Eintragungen im Klavierauszug von Ernestine Schumann-Heink in die Kritische Edition der Elektra übernommen wurden. ${ }^{58}$ Fasst man jedoch die Interpretationsleistung Bahr-Mildenburgs als Werk ,sui generis‘ und ihren Regieklavierauszug als Dokument des Entstehungsprozesses auf, so könnte dies eine Bereicherung für die Interpretationsforschung und - wie zu zeigen sein wird - für digitale Musikwissenschaft sein.

All dies kann nämlich nur sichtbar gemacht werden, wenn man diese Materialien mit digitalen Methoden erarbeiten und präsentieren würde. Da dies meines Wissens noch nie geschehen ist, ist editionswissenschaftlich daran interessant, erstmals Materialien digital zu edieren, die Theatergeschichte, verstanden als Interpretationsgeschichte, sichtbar machen können. Vorbild hierfür könnten die Arbeiten sein, die für das Detmolder Hoftheater bereits angestrengt wurden. Dabei könnte die (digitale) Transkription der Noten, wie es Agnes Seipelt formulierte, ,,mit der Auszeichnungssprache $\mathrm{MEI}^{59}$ erfolgen, einer auf XML basierten, maschinenlesbaren Sprache, mit der notierte[ ] Musik so formalisiert beschrieben werden kann, dass damit Algorithmen ausgeführt und Analysen betrieben werden können. [...] Schriftliche Anmerkungen, die das Notierte kommentieren und ergänzen, können mit MEI direkt im Notentext hinterlegt werden, indem sie als Variante bzw. als Streichung und Hinzufügung beschrieben werden. Die MEI-Codierungen sind somit mit allen Informationen versehen, die das Autograph aufzuweisen hat ${ }^{\text {" } 60}$ (vgl. Abb. 4, die beispielhaft einen Ausschnitt aus dem von Seipelt codierten Studienbuch von Anton Bruckner zeigt).

\footnotetext{
${ }^{5}$ E-Mail des Elektra-Mitherausgebers Sebastian Bolz an mich vom 5.IO.2020.

${ }^{59}$ Music Encoding Initiative, https://music-encoding.org.

${ }^{60}$ Agnes Seipelt: Digitale Edition und Harmonische Analyse mit MEI von Anton Bruckners Studienbuch. In: Brückenschläge zwischen Musikwissenschaft und Informatik. Theoretische und praktische Aspekte der Kooperation. Beiträge der Symposien zur Digitalen Musikwissenschaft Osnabrück 2018 und Paderborn 2019 im Rahmen der Jahrestagungen der Gesellschaft für Musikforschung. In Verbindung mit der Fachgruppe Digitale Musikwissenschaft hrsg. von Stefanie Acquavella-Rauch, Andreas Münzmay und Joachim Veit. Detmold 2020 (Musikwissenschaft: Aktuelle Perspektiven. 3), S. IO5-II3, hier S. Iosf.
} 


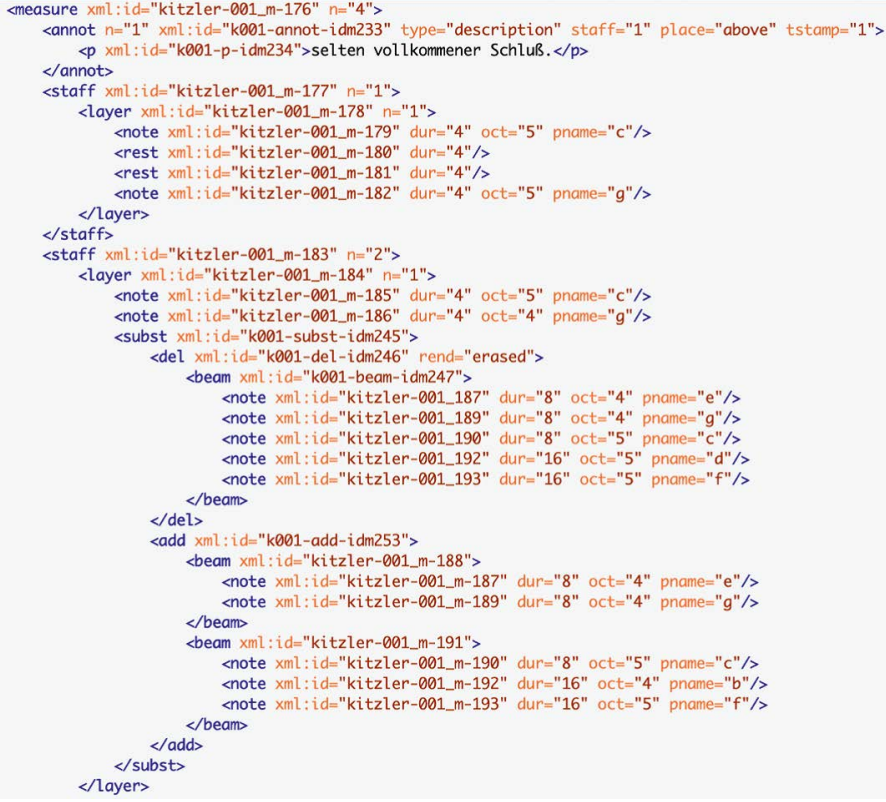

Abb. 4: Studienbuch von Anton Bruckner, Ausschnitt, digitalisiert. ${ }^{61}$

Berücksichtigt man also auch den vorhandenen Regieklavierauszug Anna BahrMildenburgs, kann auf diese Weise nicht nur die Theatergeschichte zur Elektra lebendig gemacht, sondern es können auch Querverbindungen zu anderen Materialien hergestellt werden, wie sie etwa die Kritische Ausgabe der Werke von Richard Strauss bereitstellt.

\section{Digitale Sichtbarkeit: die Musiker und die Ingenieure}

Wie oben bereits angedeutet, sollten „last but not least [auch] Klangdokumente “62 in die Edition eines Musikwerkes einbezogen werden. Auf diese Weise kann nicht nur die CD, also eine Tonaufnahme, als Werk - nämlich als Werk ,sui generis ‘ begriffen und mittels der digitalen Edition erschlossen werden, sondern es kann auch allen Beteiligten an einer Tonaufnahme zu (digitaler) Sichtbarkeit verholfen werden. Damit wird hier ebenfalls der (oben schon zitierten) Ansicht Nicholas Cooks gefolgt, der ,,auch Tonaufnahmen ein ästhetisches Daseinsrecht zu[gesteht], solange man sie auch als künstlerische ,Akte' und nicht als ,Texte“ begreift. "63 Es

\footnotetext{
${ }^{6}$ Aus Seipelt 2020 (Anm. 60), S. I06.

${ }^{62}$ Münzmay/Siegert 2019 (Anm. I), S. I5.

${ }^{63}$ Von Loesch 2019 (Anm. 7), S. II6.
} 
wird darzulegen sein, dass es sich bei CD-Produktionen in der Tat um künstlerische Akte handelt.

Während die Künstler oft namentlich und bildlich auf dem Cover einer CD erscheinen (wenn nicht doch ausschließlich mit dem Namen des Komponisten geworben wird), fehlt es den Tonmeistern und den Toningenieuren fast komplett an (digitaler) Sichtbarkeit des 'doing music' auf der CD. Sichtbar wird zudem nicht - auch nicht in Bezug auf die Künstler -, dass die Erstellung einer Tonaufnahme ein Prozess ist, der das gemeinschaftliche Zusammenwirken aller Beteiligten erfordert.

\section{a. Gruppenleistung Tonaufnahme}

Auf den ersten Blick scheint es eine Binsenweisheit zu sein, dass auch in der sogenannten ,klassischen Musik' eine befriedigende Tonproduktion ohne den durchdachten Einsatz von Aufnahmetechnik nicht möglich ist. Während jedoch bei Tonaufnahmen im Bereich der Pop- und Rockmusik nicht nur seit langem bekannt und akzeptiert ist, dass dort Aufnahmetechnik zur Erzeugung und Wiedergabe von Sound eingesetzt wird, sondern auch umfangreiche theoretische Überlegungen hierzu angestellt wurden, ${ }^{64}$ ist dies im Bereich der klassischen Musik noch nicht geschehen. Oder anders formuliert: Es gibt im Bereich der klassischen Musik noch immer keine Theorie der Musikproduktion, in der - ähnlich wie in der Filmwissenschaft - Gestaltungsprinzipien herausgearbeitet sind sowie eine wissenschaftliche Basis zu deren technischer Umsetzung und diskursiver Verhandlung zwischen den beteiligten Akteuren gelegt wurde.

Zwar wurde diese Thematik schon seit längerem in der Literatur, die von im Prozess der klassischen Musikproduktion künstlerisch und technisch beteiligten Personen verfasst wurde, behandelt ${ }^{65}$ und hat sich zum Teil auch schon in der musikwissenschaftlichen Literatur niedergeschlagen. ${ }^{66}$ In der (musikwissenschaft-

${ }^{64} \mathrm{Vgl}$. etwa Volker Smyrek: Die Geschichte des Tonmischpults. Die technische Entwicklung der Mischpulte und der Wandel der medialen Produktionsverfahren im Tonstudio von den I92oer-Jahren bis heute. Berlin 2013; Thomas Porcello: Sonic Artistry: Music, Discourse, and Technology in the Sound Recording Studio. Diss. Austin/TX 1996.

${ }^{6 s}$ Zum Beispiel Andrew Hallifax: The engineer as stylist. o. O., http://charm.cch.kcl.ac.uk/redist/pdf/ s6hallifax.pdf (gesehen 3.4.202I); Elisabeth Kemper: Realisierbarkeit und Beurteilung ästhetischer Klangkonzepte bei klassischen Musikaufnahmen. Diplomarbeit. Erich-Thienhaus-Institut, Detmold 2006.

${ }^{66}$ Zum Beispiel Hans-Joachim Maempel: Musikaufnahmen als Datenquellen der Interpretationsanalyse. In: Gemessene Interpretation. Computergestützte Aufführungsanalyse im Kreuzverhör der Disziplinen. Hrsg. von Heinz von Loesch und Stefan Weinzierl. Mainz 20II, S. I57-I72; Peter Wicke: Das Sonische in der Musik. In: PopScriptum Io, 2008: Das Sonische - Sounds zwischen Akustik und Ästhetik; Elste 1987 (Anm. 5); Bernhard Rzehulka: Abbild oder produktive Distanz? Versuch über ästhetische Bedingungen der Schallplatte. In: Gehörgänge. Zur Ästhetik der musikalischen Aufführung und ihrer technischen Reproduktion. Hrsg. von Matthias Fischer, Dietmar Holland und Bernhard Rzehulka. München I986; David Patmore, Eric Clarke: Making and hearing virtual worlds: John Culshaw and the art of record production. In: Musicae Scientiae II, 2007, H. 2, S. 269-293; Michael Chanan: Repeated Takes. A Short History of Recording and its Effects on Music. London I995; 
lichen) Öffentlichkeit jedoch und zum Beispiel in der Frage, ob es sich bei einer Tonaufnahme um ein künstlerisches Produkt (ähnlich einer professionellen Fotografie oder einem Film) handelt, gehen die Meinungen nach wie vor auseinander. ${ }^{67}$ Schon früh kommen zwar in Zeitschriften auch Aufnahmetechniker zu Wort, die von ihren Erfahrungen mit dem Grammophon berichten. ${ }^{68}$ Aber die diskursive Einbettung in einen Gesamtzusammenhang fehlt. ${ }^{69}$ Vielmehr bleibt in bisherigen Forschungsansätzen der Blick bezüglich der Tonaufnahme auf die Mediennutzung bzw. den Medienkonsum und die Auswirkungen der Medien für die Kompositionsgeschichte beschränkt. ${ }^{70}$ Die Bedingungen und Vorgänge einer Musikübertragung jedoch werden in der gegenwärtigen Forschungsdiskussion noch zu wenig berücksichtigt, insbesondere was die Ästhetik der Klangbildgestaltung betrifft. ${ }^{71}$

Man kann daher den Eindruck gewinnen, dass die Geschichte der Tonaufnahme dominiert wurde (und wird) von der Suche nach einer Wiedergabetreue, die die Zuhörer glauben lässt, das musikalische Geschehen ,wie es wirklich war' vor sich zu haben, während das Aufnahmemedium als solches, als Objekt dahinter verschwindet. Die Gründe hierfür mögen im Geniediskurs sowie im Autorschafts- und Werkdiskurs zu suchen sein, aber vermutlich auch in Marketingstrategien, Firmengeheimnissen, ${ }^{72}$ dem Natürlichkeitsdiskurs ${ }^{73}$ und sicher auch in Abgrenzungsbestrebungen zwischen dem Klassik- und dem Popmusiksektor. ${ }^{74}$ Aber untersucht ist dies nicht. Vielmehr ist festzustellen, dass es in der wissenschaftlichen Literatur und auch in der empirischen Forschung äußerst wenig Überlegungen zu einer Klangästhetik von Tonträgern gibt ${ }^{75}$ bzw. dass diese ,unterbelichtet' ist. $^{76}$

Colin Symes: Setting the Record Straight. Connecticut 2004; Mark Katz: Capturing Sound. How Technology Has Changed Music. Berkeley 2004.

${ }^{67} \mathrm{Vgl}$. Nicholas Cook: Methods for analysing recordings. In: The Cambridge Companion to Recorded Music. Hrsg. von Nicholas Cook, Eric Clarke, Daniel Leech-Wilkinson und John Rink. Cambridge 2009, S. $22 \mathrm{I}-245$.

${ }^{68}$ Zitiert in Stefan Gauß: Rille, Nadel, Trichter. Zur Kulturgeschichte von Phonograph und Grammophon in Deutschland (1900-1949). Köln 2009, passim; vgl. insbesondere das Kapitel „Die Aufnahme und das Aufgenommene", S. I66-2I6.

${ }^{69}$ Dies leisten weder Gauß 2009 (Anm. 68) noch Herbert Jüttemann: Phonographen und Grammophone. Braunschweig 1979.

${ }^{70}$ Vgl. Nils Grosch: Medienwelten. In: Geschichte der Musik im 20. Jahrhundert. I900-1925. Hrsg. von Siegfried Mauser und Matthias Schmidt. Laaber 2005, S. 205-223.

${ }^{71}$ Vgl. Maempel 20II (Anm. 66).

${ }^{72}$ Vgl. Stefan Weinzierl, Christoph Franke: „Lotte, ein Schwindel“. Geschichte und Praxis des Musikschnitts am Beispiel von Beethovens 9. Symphonie, Vortrag auf der 22. Tonmeistertagung - VDT International Convention, Hannover 2003, https://www2.ak.tu-berlin.de/ akgroup/ak_pub/2002/ Weinzierl_2002_Lotte_ein_Schwindel_Geschichte_und_Praxis_des_Musikschnitts.pdf, S. 3 .

${ }^{73}$ Vgl. Michael Harenberg: Leiblichkeit und das Körperspiel der Maschinen. In: Spiel (mit) der Maschine. Hrsg. von Marion Saxer. Bielefeld 20I6, S. 227-244.

${ }^{74}$ Vgl. Martin Pfleiderer: Musikanalyse in der Popmusikforschung. Ziele, Ansätze, Methoden. In: PopMusicology. Perspektiven der Popmusikwissenschaft. Hrsg. von Christian Bielefeldt, Udo Dahmen und Rolf Großmann. Bielefeld 2008, S. I53-I7I.

${ }^{75}$ Vgl. Kemper 2006 (Anm. 65), S. 6-9

${ }^{76}$ Vgl. Maempel 20II (Anm. 66), S. I60. 
Die oben erwähnen Stimmen in der Literatur könnte man in dem schon erwähnten Wort von Hans-Joachim Maempel zusammenfassen: Es ist bekannt, dass es sich bei der Tonaufnahme um eine „Gruppenleistung“"77 handelt. Jedoch wurde bisher von keiner Seite gefragt, wie diese denn genau aussieht bzw. zustande kommt, und es wurde nicht gefragt, welche Folgerungen dies etwa für den Autorschafts- und den Werkbegriff haben könnte.

Dies mag auch daran liegen, dass bislang keine Methode entwickelt wurde, um das Tun der Beteiligten und die Ergebnisse auch in der klassischen Musikproduktion angemessen zu beschreiben. Auch wenn die Begrifflichkeiten zur Beschreibung eines Klangereignisses seit längerer Zeit etabliert sind und von Technikern bei ihrer Arbeit auch verwendet werden, ${ }^{78}$ ist zum einen unklar, ob dieses Vokabular auch von den übrigen Beteiligten benutzt wird (oder ein anderes). Zum anderen ist bis jetzt unerforscht, wie sich die verschiedenen Beteiligten unter- und miteinander über ihre Ziele, Wünsche und ästhetischen/künstlerischen Absichten sowie nicht zuletzt über den jeweiligen räumlichen Klangeindruck verständigen, ${ }^{79}$ damit am Ende einer Aufnahmesitzung ein für alle befriedigendes klangliches Ergebnis steht. $^{80}$

Mit meinem seit März 2019 an der TU Berlin/Audiokommunikation laufenden DFG-Forschungsprojekt „Das Tonstudio als diskursiver Raum“ habe ich mich auf den Weg gemacht, um zu den vorstehend skizzierten Fragen Grundlagen zu erarbeiten. Hierfür habe ich vielerlei CD-Produktionen als Hospitantin im Tonstudio begleitet. Im Mittelpunkt stand hierbei einerseits, die Kommunikation der Ingenieure untereinander und die Kommunikation des Tonmeisters mit den Musikern zu beobachten. Andererseits lautete die Fragestellung, welche technischen Folgerungen die Ingenieure aus diesen Kommunikationssituationen gezogen haben. ${ }^{81}$ Ergänzend wurden zahlreiche Interviews und Gespräche mit beteiligten Musikern (Solisten, Dirigenten, Chorsängern, Instrumentalisten, Ensembleleitern) sowie mit Tonmeistern und Toningenieuren geführt. Die gesamte Kommunikation wurde auf

77 Maempel 2007 (Anm. 6), S. I63.

${ }^{78}$ Vgl. Kemper 2006 (Anm. 65); Susanne Binas-Preisendörfer: Rau, süßlich, transparent oder dumpfSound als eine ästhetische Kategorie populärer Musikformen. Annäherung an einen populären Begriff. In: PopScriptum Io, 2008: Das Sonische - Sounds zwischen Akustik und Ästhetik; Stefan Weinzierl, Hans-Joachim Maempel: Zur Erklärbarkeit der Qualitäten musikalischer Interpretationen durch akustische Signalmaße. In: Gemessene Interpretation 2011 (Anm. 66), S. 213-236.

${ }^{79}$ Dies ist als ein Desiderat beschrieben bei Stefan Weinzierl: Wirklich dabei sein. In: forschung. Das Magazin der Deutschen Forschungsgemeinschaft 37, 2012, H. 3, S. 4-9.

${ }^{80}$ Auf die Schwierigkeiten, sich zu verständigen, weist etwa hin Andrew Hallifax: The Classical Musician's Recording Handbook. London 2004, S. 39: "music and sounds are capricious and tricky subjects to describe without causing misunderstandings and without views seeming to be polarised."

${ }^{81}$ Hierbei lag ein besonderes Augenmerk auf den verwendeten Mikrofonen als 'tool' im Aufnahmeprozess. Eine Analysemöglichkeit ergibt sich auf der Basis einer Artefaktanalyse. Vgl. dazu Karin Martensen: Mikrophon und Habitus. Überlegungen zum Mikrophongebrauch in der klassischen Tonaufnahme. In: Rundfunk und Geschichte. Objektgeschichten des Rundfunks 46, 2020, Nr. I-2, S. $47-58$. 
Band aufgezeichnet und anschließend transkribiert. Überdies wurden verschiedene Musikzeitschriften unterschiedlicher Jahrgänge mit der Frage analysiert, wie der öffentliche Diskurs über Aufnahmeparameter dort abgebildet wird; dies geschah mithilfe von MaxQDA.

Indem die vorstehend skizzierten Daten schriftlich erfasst und ausgewertet wurden, kann erstmals gezeigt werden, dass Tonaufnahme kein (diskursiver) Raum ist, der außerhalb von Gesellschaft steht. Zum einen geht es hier um die Aushandlung von Wissen und Erfahrung sowie um Machtstrukturen wie in jeder anderen Kommunikation auch. Zum anderen hat Tonaufnahme am gesamtkulturellen Prozess teil, und das macht sie auch für übergeordnete Betrachtungen interessant: Hier wird nicht nur die ,Wahrheit‘ des Kunstwerks, sondern auch die Entstehung von Klang und die Authentizität des Künstlers verhandelt - oder eigentlich müsste man sogar sagen: Klang und Authentizität werden hierdurch hergestellt. Wir können hier also ,in nuce ' beobachten, wie 'doing technology' vor sich geht, wie also das Verhältnis zwischen Mensch und Technik tatsächlich ausgehandelt wird.

\section{b. Weitere analytische und praktische Ansätze}

Ich möchte vorschlagen, noch einen Schritt weiterzugehen und sich den in der Pop- und Rockmusik schon länger vorhandenen bzw. jetzt neu entwickelten methodisch-analytischen ${ }^{82}$ und praktischen Ansätzen zuzuwenden. Diese und ihre Relevanz für den Autorschafts- und den Werkbegriff seien nachstehend kurz skizziert.

Mit dem Soundbegriff konnten in den letzten Jahren bedeutende Fortschritte in der Popmusikforschung in dem Bemühen erzielt werden, das ,Werk' eines Sängers bzw. einer Sängerin oder von Popmusikgruppen dieses Genres zu beschreiben, nachdem sich eine Herangehensweise an diese Musik in der Art, wie sie in der sogenannten ,Klassischen' Musik üblich ist, als unzureichend erwiesen hatte. ${ }^{8_{3}}$ Dieser ,Sound“, „,dessen Extensionen nach Einschätzung der Musikwissenschaft ästhetische, akustische, technologische, ökonomische, soziale sowie psychologische Komponenten enthält“, kann nicht auf das gemeinsame Material ,Ton“ zurückgeführt und auch nicht mit den Komponenten Melodik, Harmonik, Rhythmik und Form beschrieben werden. Es gilt daher, schreibt Dietrich Helms, sich vom Paradigma Ton zu lösen, um auch solche Musik beschreibbar zu machen. ${ }^{84}$

\footnotetext{
${ }^{82} \mathrm{Zu}$ diesen s. beispielhaft Peter Wicke: Popmusik in der Analyse. In: Acta Musicologica 75, 2003, S. I07-I26.

${ }^{83}$ Siehe etwa Martin Pfleiderer: Sound. Anmerkungen zu einem populären Begriff. In: Pop Sounds. Klangtexturen in der Pop- und Rockmusik. Basics - Stories - Tracks. Hrsg. von Thomas Phleps und Ralf von Appen. Bielefeld 2003 (Texte zur populären Musik. I), S. 19-29. Zugleich zeigt der genannte Sammelband die große Bandbreite der Definitionen, die derzeit in der Popularmusikforschung für den Begriff des, Sounds' verwendet werden.

${ }^{84}$ Dietrich Helms: Auf der Suche nach einem neuen Paradigma: Vom System Ton zum System Sound. In: Pop Sounds 2003 (Anm. 83), S. 197-228, hier S. I99.
} 
Es soll hier jedoch nicht primär um Fragen der Popmusikforschung gehen, auch wenn diese - wie im Folgenden deutlich werden wird - als für die Historische Musikwissenschaft außerordentlich bereichernd angesehen werden können. Es fragt sich für die letztgenannte Disziplin nämlich, ob nicht auch die umgekehrte Überlegung angestellt werden sollte: ob nämlich mit den im vorigen Absatz beschriebenen Elementen des ,Paradigmas Ton“ die sogenannte klassische Musik überhaupt hinreichend genau beschrieben werden kann, gerade wenn man sich darüber klar wird, dass auch diese ästhetische, akustische, technologische, ökonomische, soziale sowie psychologische Komponenten enthält. Mir scheint, dass dies nicht der Fall ist, sondern dass etwa zur Beschreibung des gesamten Phänomens ,Gesang' auf alternative Begrifflichkeiten zurückgegriffen werden muss. ${ }^{85}$ Dies ließe sich mit dem aus dem Bereich der U-Musik bekannten Sound-Begriff bewerkstelligen, wenn dieser auf den Bereich , $E^{‘}$ übertragen würde. Die Stimme ist nicht ein Medium ,sui generis', wie Ludwig Pfeiffer meint, ${ }^{86}$ sondern entsteht im Körper des Sängers, und zwar abhängig etwa von seiner Ausbildung, seinen ästhetischen Vorstellungen und seinen körperlichen Voraussetzungen. Somit ist sie als ,Werk' des Sängers zu verstehen, das in seiner medialen Außendarstellung auch von ,Soundtechnologien“ abhängt.

Aber wie kann ein Sound (und zwar nicht nur in Bezug auf die Singstimme, sondern für alle Instrumente) und damit das Werk eines Künstlers tatsächlich sichtbar gemacht werden, wenn man sich dabei nicht auf sprachliche Beschreibungen oder auf Einzelaspekte beschränken will, die mit dem Sonic Visualiser beschrieben werden können? Und wie können die ,Soundtechnologien“ sichtbar gemacht werden, hier verstanden als der Schaffensprozess zwischen Tonmeister und Künstlern bei einer Tonaufnahme? Ein Blick in die Pop- und Rockmusikforschung zeigt, dass dort den Interpreten und den Produzenten anerkanntermaßen überragende Bedeutung für die Entstehung und Vermarktung des Musikwerkes zukommt. ${ }^{87}$ Es wird zu zeigen sein, dass man dort, also in der Pop- und Rockmusikforschung, auch diesbezüglich für unser Fach lohnenswerte ,Anleihen“ machen kann.

Wie Stefanie Acquavella-Rauch in ihrem spannenden Pilotprojekt zeigen konnte, ${ }^{88}$ kann dieser Schaffensprozess bei einer Tonaufnahme mit digitalen Methoden nachvollziehbar gemacht werden. Auf der Website des Projekts heißt es:

\footnotetext{
${ }^{85}$ Vgl. Karin Martensen: Der Soundbegriff - eine Methode zur Beschreibung des Phänomens „,Operngesangsstimme"? In: Archiv für Musikwissenschaft 74, 20I7, S. I40-I59.

${ }^{86}$ Ludwig Pfeiffer: Operngesang und Medientheorie. In: Stimme. Annäherung an ein Phänomen. Hrsg. von Doris Kolesch und Sybille Krämer. Frankfurt/Main 2006, S. 65-84.

${ }^{87}$ Siehe dazu nur Alfred Smudits: A Journey into sound. Zur Geschichte der Musikproduktion, der Produzenten und der Sounds. In: Pop Sounds 2003 (Anm. 83), S. 65-94.

${ }^{88} \mathrm{Vgl}$. https://schaffensprozess-2-O.uni-mainz.de/ (gesehen 28.9.2020). Vgl. hierzu auch Stefanie Acquavella-Rauch: Musikalische Schaffensprozesse 2.0 - Inkorporation audiovisueller Medien der populären Musik in Methoden der digitalen Edition. In: Brückenschläge zwischen Musikwissenschaft und Informatik 2020 (Anm. 60), S. II5-I22.
} 
Das Projekt ,Musikalische Schaffensprozesse 2.0 - Inkorporation audiovisueller Medien der populären Musik in Methoden der digitalen Edition' hat zum Ziel, bisher nicht erfassbare Dimensionen musikalischer Entstehungsprozesse dokumentierbar, edierbar und damit untersuchbar zu machen. Sämtliche bisherige Ansätze der historischen Musikwissenschaft und der digitalen Musikedition setzen voraus, dass Musik und Klang schriftlich notiert werden, was mit Hilfe des Projekts entscheidend um eine mediale Dimension des Komponierens erweitert werden soll.

Hierfür wurde die Melodic Hardcore Band „Close to the Distance“ in Proben begleitet und deren gruppenbezogener Schaffensprozess nicht nur mit MaxQDA, sondern auch mit den Methoden der digitalen Edition erfasst. Acquavella-Rauch kann zeigen, dass ,mit Hilfe der angewandten, sich von den etablierten Methoden der historischen Musikwissenschaft stark unterscheidenden ethnographischen Ansätze die gleichen Fragestellungen zum musikalischen Schaffensprozess verfolgt werden können, die teils zu ähnlichen und teils zu neuen Erkenntnissen führen." Insbesondere können mit digitalen Methoden auch auditive Medien inkorporiert werden, da ,„die auf Schriftlichkeit ausgerichteten Zugänge der Musikeditorik der speziellen Art der erhobenen Daten nur sehr eingeschränkt gerecht werden können. “89 Neben der Track-Segmentation, der Synchronisierung von auditiven Signalen und Noten sowie der Erkennung von gesprochenen und gesungenen Passagen handelt es sich dabei auch um das Processing von Multitrack-Aufnahmen. ${ }^{90}$

Ein anschauliches Beispiel für das Vorgehen und seine vielfältigen Möglichkeiten findet sich etwa auf der Website des Projekts Freischütz Digital:

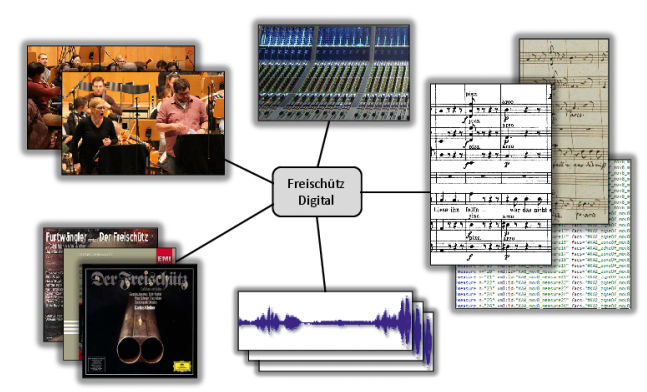

Abb. 5: „Music-related information in multiple modalities illustrated by means of the opera ,Der Freischütz' by Carl Maria von Weber"; https://freischuetz-digital.de/documentation-audio.html (gesehen 23.10.2020).

\footnotetext{
${ }^{89} \mathrm{https}: / /$ schaffensprozess-2-0.uni-mainz.de/ergebnisse.html (gesehen 23.10.2020).

${ }^{90} \mathrm{https://schaffensprozess-2-0.uni-mainz.de/digitalemusikedition.html} \mathrm{(gesehen} \mathrm{23.10.2020).}$
} 


\section{c. Datenbasis: Hospitationen}

Dieser Ansatz kann - ebenso wie der Soundbegriff - mit Gewinn auf den Bereich ,klassische Musik، übertragen werden, um den dort stattfindenden Schaffensprozess in der Zusammenarbeit von Musikern und Ingenieuren (Tonmeister und Toningenieur) bei der Produktion einer CD sichtbar zu machen. Auf der Basis meiner Forschungsdaten aus dem oben erwähnten DFG-Projekt „Das Tonstudio als diskursiver Raum“91 (teilnehmende Beobachtung, Interviews, Transkripte von Aufnahmeprozessen, Auswertungen mit MaxQDA) kann gezeigt werden, dass diese Schaffensprozesse im gemeinschaftlichen Zusammenwirken von Künstlern und Ingenieuren auch in der klassischen Musik zu finden sind. ${ }^{92}$

In der Diskussion der Musiker mit dem Tonmeister wird deutlich, dass - gemeinsam mit diesem - ein Schaffensprozess in Bezug auf das Werk stattfindet, dessen Ergebnis dann (nach Abschluss des Digitalschnitts durch den Tonmeister) auf der veröffentlichten CD dokumentiert ist. Dass dieser Schaffensprozess nicht nur kommunikativer, sondern auch in erheblichem Umfang technischer Art ist, verdeutlicht der Austausch zwischen Tonmeister und Toningenieur. Die Ingenieure verständigen sich hierbei etwa darüber, welches klangliche Gewicht ein einzelnes Instrument oder eine Instrumentengruppe im Verhältnis zu den anderen Instrumenten haben soll und ob die Mikrofonierung ausreichend, also werkangemessen ist.

Dies mit Hilfe von digitalen Methoden aufzuarbeiten und zu präsentieren ist ein lohnendes Projekt. Editionswissenschaftlich geht es auch hierbei (ebenso wie bei der digitalen Sichtbarmachung der Interpretationsleistung; s.o.) darum, die Materialien - in diesem Fall: meine Hospitationsergebnisse und gegebenenfalls neu zu erhebende Daten - als Editionsobjekte zu begreifen. Es würde nicht nur deutlich werden, dass die Tonaufnahme ein mediales Kunstobjekt ${ }^{93}$ und damit in der Tat ein Werk , sui generis‘ ist. Eine digitale Sichtbarkeit könnte auch zur Wertschätzung der Musik bzw. der Tonaufnahme und der dort tätigen Personen beitragen.

\section{Fazit}

Während in meinem vorgenannten DFG-Projekt eine schriftliche Auswertung und Kontextualisierung von Hospitationen geschieht, würde es sich zudem lohnen, den skizzierten Ansatz von Acquavella-Rauch zu übernehmen und für eine, Tiefenboh-

\footnotetext{
${ }^{91}$ Das Tonstudio als diskursiver Raum: Theorie, ästhetisches Konzept und praktische Umsetzung; Antragstellerin: Dr. Karin Martensen (Eigene Stelle); Projektnummer 4I99II7I2; https://gepris.dfg. de/gepris/projekt/4I99II7I2 (gesehen 3I.3.202I).

${ }_{92}$ Ein ausführlicher Forschungsbericht zu diesem Projekt ist in Arbeit und wird voraussichtlich im Frühjahr 2022 publiziert werden.

${ }_{93} \mathrm{Vgl}$. hierzu auch Karin Martensen: Überlegungen und Interviews zum Einsatz von Aufnahmetechnik und zur Gruppenleistung Tonaufnahme im Bereich der Klassischen Musik. In: Acta Musicologica 2017, H. 2, S. I45-I70.
} 
rung' auch in der klassischen Musik zu nutzen. Durch eine Auswertung dieser (und neuer) Materialien mithilfe von MEI und TEI können neue Wege von ,Werk' und ,Autorschaft' auch in diesem Genre beschritten werden.

Ziel aller dieser Überlegungen ist es, den Schaffensprozess der Interpreten bzw. der Interpretinnen und ihren Einfluss auf das Werkverständnis plausibel und sichtbar $\mathrm{zu}$ machen. Es soll gezeigt werden, dass sich Interpretation in der Tat ,als künstlerische Auseinandersetzung mit einem bestimmten, als Aufführungsmaterial [...] benutzten Notentext (oder auch mehreren) darstellt". ${ }^{44}$ Und dies gilt eben nicht nur für eine Sängerin oder einen Sänger, der bzw. die ein Werk erarbeitet, sondern auch für die ,Gruppenleistung von Musikern und Ingenieuren bei einer Tonaufnahme. Auf diese Weise soll der (gemeinschaftliche) Prozess des Interpretierens verdeutlicht werden. In beiden Fällen stellt sich das Geschaffene (also die Interpretationsleistung bzw. die CD) als eigenständiges Werk, als Werk , sui generis“ dar. Auch der Schaffensprozess selbst bzw. die Materialien, die ihm zugrunde liegen, bedürfen aber der Beachtung durch die Interpretationsforschung, die hiermit angeregt werden soll.

Mit dem vorstehend skizzierten Ansatz sollen Daten, Methoden und Instrumente implementiert werden, um die Dominanz des ,Partiturwissens“ in der historischen Musikwissenschaft durch eine ,Klangwissenschaft ${ }^{`} \mathrm{zu}$ ersetzen. ${ }^{95}$ Dass Musizieren ein Tun ist und soziale Praxis, kann mit diesen neuen Möglichkeiten nicht nur behauptet, sondern auch bewiesen werden. ${ }^{96}$

\section{Abstract}

The concept of work and authorship have long been the subject of intense reflection in historical musicology. Edition projects deal with composers and their work process and have developed new approaches for this purpose. Against the background of the question of where the 'text' to be edited actually ends, this paper discusses the possibilities of making director's piano score (Regieklavierauszug) digitally visible and thus interpretable (understood as a work 'sui generis'). Furthermore, the creative process of all participants in the classical sound recording is considered in terms of its digital visibility. On the basis of my research data from the DFG project "The sound recording studio as a discoursive room" at the TU Berlin/Audiokommunikation (participant observation, interviews, transcripts of recording processes, evaluations with MaxQDA) I show that these creative processes in the collaborative interaction of artists and engineers can also be found in classical music. By evaluating these (and other) materials with the help of MEI and TEI, new ways of 'work' and 'authorship' are also explored in this genre. Ultimately, this should also establish the sound recording as a 'sui generis' work.

\footnotetext{
${ }_{94}$ Münzmay/Siegert 2019 (Anm. I), S. 25.

${ }^{95}$ Vgl. Elste 1987 (Anm. 5) und die Ausführungen hierzu oben.

${ }^{96}$ Für anregende Diskussionen und hilfreichen Rat bei der Entstehung dieses Aufsatzes bedanke ich mich sehr herzlich bei meinem Kollegen Kilian Sprau (Universität der Künste Berlin).
} 